\title{
画HAD
}

DOI: http://doi.org/10.22585/hospdomic.v2i2.37

\section{Drenaje peritoneal permanente en paciente con mesotelioma metástasico: ganancia en calidad de vida en paciente oncológica}

Permanent peritoneal drainage in patient with metasstasic mesothelioma: gain in quality of life in oncological patient

Andrea Illán', Laura Quintana', Manuela Chiquero², Rocío Gallego³, Ángel Domínguez $^{2}$, Jonathan Aires', Sergio Moyano ${ }^{4}$, Rubén Alonso', Selene Illán'

1. Hospital San Pedro de Alcántara, Servicio de Oncología Médica, Cáceres, España.

2. Hospital San Pedro de Alcántara, Servicio de Medicina, Cáceres, España.

3. Hospital San Pedro de Alcántara, Servicio de Neumología, Cáceres, España.

4. Hospital San Pedro de Alcántara, Servicio de Radiodiagnóstico, Cáceres, España..

Correspondencia/Correspondence

Andrea llán Varella

Avenida de los Pilares, 9-1 ${ }^{\circ} \mathrm{C} 10003$ Cáceres

634571707

andrea88med@yahoo.es

Recibido/Received

29.01.2018

Aceptado/Accepted

05.02.2018
Conflicto de Intereses/Competing interest

Los autores no presentan conflicto de intereses

Cómo CitAR ESTE tRABAjo | HOW TO CITE tHIS PAPER

Illán A. Quintana L. Chiquero M. Gallego R. Domínguez A. Aires J. Moyano S. Alonso R. Illán S. Drenaje peritoneal permanente en paciente con mesotelioma metástasico: ganancia en calidad de vida en paciente oncológica. Hosp Domic. 201 8;2(2):79-82 


\section{RESUMEN}

Presentamos el caso de una paciente, mujer de 63 años diagnosticada de mesotelioma epiteliode estadío IV. Durante la evolución progresiva de su enfermedad, fue precisando paracentesis evacuadoras frecuentes con correspondientes visitas habituales al servicio de Urgencias. Dada la situación, en Septiembre de 2016 se procedió a colocación por parte del Servicio de Radiología y Neumología de catéter Pleur $X ®$ en cavidad peritoneal, a nivel de fosa ilíaca derecha. La paciente presentó buena tolerancia, sin necesidad de más paracentesis en urgencias, y con gran beneficio en su calidad de vida.

Palabras clave: Mesotelioma; Paracentesis; Catéteres; Catéteres de Permanencia.

\section{ABSTRACT}

We present the case of a 63-year-old female patient diagnosed with stage IV epithelial mesothelioma. During the progressive evolution of his illness, frequent evacuation paracentesis was required with regular visits to the Emergency Department. Given the situation, in September 2016, the Radiology and Pneumology Service of the Pleur $X{ }^{\circledR}$ catheter was placed in the peritoneal cavity, at the level of the right iliac fossa. The patient presented good tolerance, without needing more paracentesis in the emergency room, and with great benefit in their quality of life.

Keywords: Mesothelioma; Paracentesis; Catheters; Catheters, Indwelling. 


\section{INTRODUCCIÓN}

La ascitis maligna, una entidad frecuente en pacientes oncológicos, representa múltiples complicaciones, entre ellas, la necesidad de paracentesis evacuadoras repetidas, con el consecuente compromiso relevante de su calidad de vida. Presentamos el caso de una paciente diagnosticada de mesotelioma maligno metastásico con ascitis maligna, a la que se decidió colocar drenaje peritoneal tipo Pleur $x^{\circledR}$, con eliminación de la necesidad de paracentesis periódicas, posibilidad de ser manipulado por la propia paciente y gran ganancia en calidad de vida.

\section{Desarrollo de la experiencia clínica:}

Presentamos el caso de una paciente mujer de 63 años que inició estudio y seguimiento por medicina interna en el año 2006 por ascitis. Tras estudio diagnóstico, Fue diagnosticada en base a biopsia pleural de mesotelioma epiteliode. Fue derivada a consultas externas de Oncología Médica. Recibió 6 ciclos Carboplatino-Pemetrexed (último ciclo en junio 2013). Desde Junio de 2014 hasta la actualidad, recibió otras líneas de tratamiento citostático por progresos fundamentalmente a nivel pleuro-peritoneal: Retratamiento con Carboplatino-Pemetrexed, Gemcitabina, y Vinorelbina (tratamiento que sigue en el presente).

La paciente fue precisando paracentesis evacuadoras frecuentes. Dada la situación, en Septiembre de 2016 se procedió a colocación por parte del Servicio de Radiología y Neumología de catéter PleurX ${ }^{\circledR}$ (Figura 1) en cavidad peritoneal, a nivel de fosa ilíaca derecha. La técnica se realizó bajo control ecográfico. No se desarrollaron complicaciones inmediatas.

Figura 1. Drenaje peritoneal PleurX®

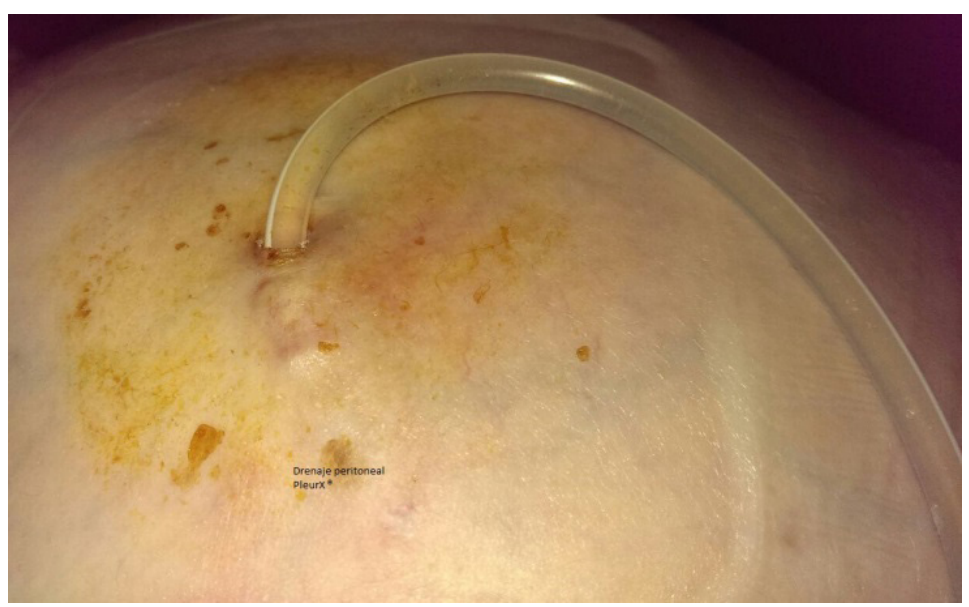

Dos semanas después de su colocación la paciente presentó fiebre y en el análisis de líquido ascítico se observó bacteriascitis sin signos de peritonitis bacteriana espontánea (PBE), y con cultivo del mismo negativo. La paciente completó tratamiento antibiótico con buena respuesta clínicoanalítica. Actualmente, la paciente extrae a diario $500 \mathrm{cc}$ de líquido ascítico, sin necesidad de más paracentesis regladas en el servicio de Urgencias. La reposición de albúmina así como a continuación de los ciclos de quimioterapia, se realizan en Hospital de Día. 
La presentación del caso clínico implica interés clínico al representar el único caso descrito en la literatura científica de beneficio en calidad de vida por la colocación de catéter pleural a nivel peritoneal para tratamiento de la ascitis maligna de repetición.

Saiz-Mendigurena et al (1), en 2010, describían su experiencia con el empleo de drenaje permanente tunelizado Pleur $X^{\circledR}$ en la ascitis maligna (1). Emplearon guía por ecografía y anestesia local. La tolerancia al procedimiento fue adecuada. El volumen de drenaje semanal osciló entre 1 y 7 litros de líquido ascítico.

Clásicamente, la supervivencia estimada de pacientes con ascitis maligna rondaba entre los 2 y 6 meses, aunque esta cifra se ha venido modificando con los avances en el tratamiento sistémico citostático (2). El manejo de la ascitis maligna puede incluir diuréticos de asa (Furosemida) y ahorradores de potasio (Espirinolactona), aunque en la mayor parte de los casos será necesaria la realización de paracentesis evacuadoras para alivio de síntomas asociados (dolor abdominal, distensión abdominal y disnea, entre otros) de tipo paliativo (3) (en centros hospitalarios y a domicilio) y reposición de albúmina posterior.

El desarrollo de técnicas intervencionistas que faciliten la evacuación de la ascitis maligna ha supuesto una mejora de la calidad de vida de estos pacientes. Las derivaciones peritoneo-venosas (4) (shunts de LeVeen y de Denver) y las válvulas peritoneo-auriculares, son técnicas quirúrgicas para el manejo paliativo de la ascitis maligna a largo plazo. Courtney et al (5), aportaron que el uso del catéter peritoneal Pleur ${ }^{\circledR}$ en paciente oncológico terminal implica una mejoría del «disconfort» secundaria a ascitis y que se asocia a tasas bajas de eventos adversos serios y fallo del catéter.

\section{CONCLUSIONES}

La necesidad de paracentesis evacuadoras en el servicio de Urgencias por ascitis maligna, resulta un hecho que implica gran detrimento en la calidad de vida en el paciente oncológico. La colocación de catéter permanente tunelizado a nivel peritoneal representa una herramienta de fácil autoaprendizaje, gran efectividad en domicilio y disponible en nuestro medio.

\section{BIBLIOGRAFÍA}

1. Saiz-Mendigurena R, Gómez-Ayechu M, Noguera JJ, García-Lallana A, Marginet C, Cano D, et al. Drenaje permanente tunelizado de la ascitis maligna: experiencia inicial con el catéter PleurX. Radiología. 2010;52(6):541-5. DOI: 10.1016/j.rx.2010.06.005; PMID: 20863540

2. Mirabelli D, Roberti S, Gangemi M, Rosato R, Ricceri F, Merler E, et al. Survival of peritoneal malignant mesothelioma in Italy: A population-based study. Int J Cancer. 2009;124(1):194-200. DOI: 10.1002/ijc.23866; PMID: 18792099

3. Mott FE. Mesothelioma: a review. Ochsner J. 2012;12(1):70-9. PMID: 22438785

4. Rosenberg S, Courtney A, Nemcek AA Jr, Omary RA. Comparison of percutaneous management techniques for recurrent malignant ascites. J Vasc Interv Radiol. 2004;15(10):1129-31. DOI: 10.1097/01.RVI.0000136828.42612.B4; PMID: 15466800

5. Courtney A, Nemcek AA, Rosenberg S, Tutton S, Darcy M, Gordon G. Prospective evaluation of the PleurX catheter when used to treat recurrent ascites associated with malignancy. J Vasc Interv Radiol. 2008;19(12):1723-31. DOI: 10.1016/j.jvir.2008.09.002; PMID: 18951041 